1

2

3

4

5

6

7

8

9

\title{
Quantitative NMR imaging study of the cooking of Japonica and
}

\section{Indica rice}

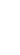

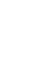

10

\author{
Midori Kasai $^{\text {a,b, } *}$, Andrew R. Lewis ${ }^{c}$, Sonoko Ayabe ${ }^{\text {d }}$, Keiko Hatae ${ }^{\text {e }}$, Colin A. Fyfe ${ }^{\text {b }}$
}

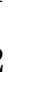

13

\footnotetext{
${ }^{a}$ Faculty of Human Life and Environmental Science, Ochanomizu University, 2-1-1 Otsuka, Bunkyo-ku, Tokyo 112-8610, Japan

${ }^{\mathrm{b}}$ Department of Chemistry, The University of British Columbia, 2036 Main Mall, Vancouver, BC Canada V6T 1Z1

${ }^{\mathrm{c}}$ Department of Chemistry, Simon Fraser University, 8888 University Drive, Burnaby, BC Canada V5A 1S6

\author{
${ }^{\mathrm{d}}$ Department of Health and Nutrition, Takasaki University of Health and Welfare, Takasaki, Gunma 370-0033, Japan
}

${ }^{\mathrm{e}}$ Department of Human Life and Environment, Wayo Women's University, Ichikawa Chiba 272-8533, Japan
}

8

Submitted to Food Research International 


\begin{abstract}
NMR imaging was used to follow changes in the water content and distribution during the cooking of Nipponbare (Japonica), Khao Dawk Mali (Indica) and High Amylose (Indica) rice. Samples were cooked in closed glass vials for various times, quenched to stop cooking and then investigated using two-dimensional multi-echo ${ }^{1} \mathrm{H}$ imaging experiments at $20{ }^{\circ} \mathrm{C}$. Images calculated from the first echo revealed changes in the water content of the rice grains. $T_{2}$ images calculated using the first 16 echoes were converted into quantitatively reliable contour maps of the water concentration using an empirical $T_{2}$ vs. water content calibration determined from a series of water/rice starch mixtures. Considerable differences in the rate of water uptake and diffusion, the average water contents and the proportions of under-, optimally- and over-cooked rice were observed for the three rice types studied.
\end{abstract}

\title{
Keywords
}

NMR imaging; $\mathrm{T}_{2}$; water content; Indica rice; Japonica rice; cooking 


\section{Introduction}

Originally native to South Asia and certain parts of Africa, rice is now a staple food for a large part of the world's human population. The two major varieties of rice are Indica and Japonica and there are several cultivars of these types. Differences between these rice varieties include the length of the grains, the taste, and the composition of the rice flour. Japonica grains are usually shorter than Indica grains and it is well known that the hardness (firmness) and texture after cooking is quite different.

Rice cooking is essentially the reaction of starch with water at elevated temperatures. As heating proceeds the starch granules within the rice grain absorb water, swell and then gelatinize with increasing temperature. At this point the granules break apart and the starch becomes gelatinized. The starch in rice is a mixture of amylose and amylopectin molecules. Amylose is a long, straight chain molecule while amylopectin is a highly branched molecule that makes the rice sticky when it is released as a result of cooking. In general, long grain rices are rich in amylose and less sticky than short grain rices which contain more amylopectin.

The properties of cooked rice vary depending on the relative ratio of amylose and amylopectin. For example, when fully cooked, long grain rices tend to remain as separate grains since they are less sticky than the short grain rices. In the present study we investigated the cooking of Nipponbare (a Japonica rice), Khao Dawk Mali rice and High Amylose rice (both Indica cultivars). Nipponbare is a short grain rice, while both Khao Dawk Mali rice and High Amylose rice are long grain rices (Fig. 1). Nipponbare and Khao Dawk Mali have similar apparent amylose contents and firmness when fully cooked but Nipponbare rice is stickier than Khao Dawk Mali rice. The texture of cooked High Amylose rice is completely different, being much firmer and less sticky than Nipponbare.

To determine the optimal cooking conditions for each type of rice it is desirable to be able to detect differences in the water absorption into the rice grains during cooking. Ideally it should also be possible to measure the water content quantitatively at various points within the individual grains in order to map the water distribution. ${ }^{1} \mathrm{H}$ NMR imaging is a technique ideally suited to this task because it detects the protons of the water molecules directly, generates images with good spatial resolution, and is non-invasive. Most importantly the relative intensities of the pixels comprising the images can be rendered quantitatively reliable so long as appropriate protocols are used to acquire and process the data. 
To date there have been several NMR imaging investigations of cooked rice that report various changes in the physical structure and moisture distribution within rice grains during or after cooking (Nakazawa et al., 1978, Horigane et al., 1999, Takeuchi et al., 1997a, Takeuchi et al., 1997b, Mohoric et al., 2004). Recently we developed a non-invasive proton $\left({ }^{1} \mathrm{H}\right)$ NMR imaging protocol for quantitatively investigating the cooking process of rice. (Kasai et al., 2005) In this method two-dimensional images generated from the first echo of the multi-echo experiments provide easy to interpret pictures showing a slice through the rice grains which allows the absorption of water to be followed during the cooking process. The slices are selected by applying appropriate combinations of magnetic field gradients during the imaging experiments.

The transverse relaxation time $T_{2}$ of the water molecules is sensitive to the local environment and thus $T_{2}$ is affected by the concentration of starch. Proton $T_{2}$ images can be calculated from the MSME echo trains by fitting the intensity changes for each pixel as a function of the total time to echo. Using an empirical relationship between $T_{2}$ and water content established from a series of water/rice starch mixtures of known composition, the $T_{2}$ images can be converted into quantitatively reliable "contour maps" which provide the water content at every point within the imaged slice through the rice grains. The degree of cooking can also be determined based on the water content.

Previous application of this NMR imaging protocol to a Japonica rice (Nipponbare) demonstrated the usefulness of the technique and validated its quantitative reliability (Kasai et al., 2005). Here we report results from a further investigation of the cooking of the Nipponbare rice studied previously, and of two Indica rices (Khao Dawk Mali and High Amylose) using the same experimental protocol.

\section{Materials and Methods}

\subsection{Samples}

90\% milled Japonica rice (Oryza sativa L. Japonica cv. Nipponbare) and Indica rice (Oryza sativa L. var. Indica cv. Khao Dawk Mali 105, and High Amylose rice) were used (Fig. 1). The apparent amylose contents measured by the method of Juliano et al (1981) were 16.95\%, 16.77\% and $27.64 \%$ respectively. The cooking method was the same as described in our previous paper (Kasai et al., 2005). $1 \mathrm{~g}$ of the milled rice was put in a screw-top vial ( $\varnothing 15 \mathrm{~mm}, \mathrm{H} 45 \mathrm{~mm}$ ) with 
distilled water and closed tightly. The ratio of water to rice was $1.9(\mathrm{w} / \mathrm{w})$, which is the standard value for Indica rice (Nishimura et al., 1997). We used this ratio to compare the Japonica and Indica rices although the standard value for Japonica rice is $1.4(\mathrm{w} / \mathrm{w})$ (Kasai et al., 2005). After the rice had soaked for $1 \mathrm{~h}$ at room temperature, the sample vials were heated in a water-filled beaker using a hot plate (Fisher Thermix). The temperature of the water in the beaker was measured using a K-type thermocouple and the temperature of the rice samples cooked this way closely track that of the water bath (Kasai et al., 2005). The samples were removed from the water bath after cooking times of $8,10,12,15,20$ and $30 \mathrm{~min}$ and immediately cooled in $15{ }^{\circ} \mathrm{C}$ water for 1.5 min to quench the cooking. The time to boiling was kept constant and was very close to $12 \mathrm{~min}$. Samples were investigated by NMR imaging at $20{ }^{\circ} \mathrm{C}$ within $30 \mathrm{~min}$ of quenching. A sealed glass capillary $(\varnothing 1.5 \mathrm{~mm})$ containing distilled water was placed in each vial prior to commencing imaging to provide a reliable intensity reference. This allowed consistent image intensities to be obtained.

\subsection{NMR imaging}

A Bruker Avance DRX360 NMR spectrometer equipped with microscopic imaging capabilities was used to acquire the ${ }^{1} \mathrm{H}$ images. Sample vials were centered inside the $20-\mathrm{mm}$ diameter coil of a Bruker Micro-2.5 imaging probe and the water signal was set on resonance (ca. $360 \mathrm{MHz}$ ) for each sample. Gradient echo fast imaging (GEFI) (Paravision 1996a) was used to determine the optimal position of the single 1-mm thick slice selected for the quantitative multi-slice multi-echo (MSME) (Paravision 1996b) imaging experiments. $500 \mu$ s sinc-3 pulses were used for both the excitation and refocusing pulses in the MSME experiments. 64 echoes were recorded for a single slice with the time between echoes (TE) set to $4.5 \mathrm{~ms}$ and the recovery time between scans (TR) was $16 \mathrm{~s}$. The field of view was fixed at $16 \mathrm{~mm} \times 16 \mathrm{~mm}$ and a matrix size of $128 \times 128$ was used, producing image voxel dimensions of $0.125 \times 0.125 \times 1 \mathrm{~mm}$ (voxel volume $=0.0156 \mathrm{~mm}^{3}$ ). The acquisition time for a MSME data set was $34 \mathrm{~min} . T_{2}$ images were calculated using the first 16 echoes with the Paravision software (Paravision 1996c).

\subsection{Calculation of water content and degree of cooking}

The water concentration was determined for each pixel in the $T_{2}$ images using the empirical relationship reported in our previous paper (Kasai et al., 2005): 
where $W$ is the water content in $\mathrm{g} / \mathrm{g}$ and $T_{2}$ has units of $\mathrm{ms}$. This equation was derived from fitting the $T_{2}$ values measured for a series of water/rice starch mixtures of known composition and is valid for water contents from 0.48 to $0.93 \mathrm{~g} / \mathrm{g}$ at $20^{\circ} \mathrm{C}$.

Because "cooking quality" is a subjective term, it is better to report the "degree of cooking' which can be defined based on the water content of the rice grains. In accordance with our previous study, the degree of cooking was classified into three categories; under-cooked, optimally-cooked and over-cooked. The range of $T_{2}$ values and corresponding water contents (in $\mathrm{g} / \mathrm{g})$ from the $T_{2}$ calibration for these three categories were $10 \leq T_{2}<17 \mathrm{~ms}(0.48 \leq W<0.6), 17$ $\leq T_{2} \leq 38 \mathrm{~ms}(0.6 \leq W \leq 0.8)$, and $38<T_{2} \leq 63 \mathrm{~ms}(0.8<W \leq 0.93)$, respectively.

A C-language computer program was written to read the binary $T_{2}$ image data files generated by the Paravision software, apply eq 1 and create text files containing the $T_{2}$ data, the water content data, and the number of pixels corresponding to under-, optimally-, or over-cooked rice. Pixels having $T_{2}$ values outside the valid range were assigned a water content value of zero to ensure the average water contents within the rice grains were correctly determined. The average water content was calculated for each rice sample using the total number of pixels in an image known to fall within rice grains (determined manually from the corresponding first echo image) and included the water present in the raw rice before soaking. The $T_{2}$ and water content data files were imported into Origin (Origin) to generate the 2-D and 3-D plots. 


\section{Results and discussion}

\subsection{Water absorption - first echo images}

8-bit grayscale images generated from the first echoes of the MSME experiments for the three types of rice at different cooking times are presented in the left columns of Figs. 2-4. In these images, white pixels correspond to bulk or "liquid" water, black pixels to water contents of $0.4 \mathrm{~g} / \mathrm{g}$ or less, and lighter gray pixels correspond to regions having higher water contents than the darker gray pixels. Each pixel in these images shows a signal from the water protons whose measured intensity reflects the water content in the corresponding rice voxel. Images calculated from the first echo (TE of $4.5 \mathrm{~ms}$ ) provide the most reliable direct detection of water within the rice because the signals have decayed the least due to $T_{2}$ relaxation.

The small black-ringed white circles in these images correspond to the water-filled capillary added to the vials after cooking. The number of rice grains (and their positions and orientations) varies within a time-series of images because the NMR images were obtained for different samples. The greater length of Indica rice grains means that they preferentially aligned parallel to the vial walls (i.e. perpendicular to the plane of the images). More grains are therefore observed "end on" in the images of Khao Dawk Mali and High Amylose than for Nipponbare.

When the cooking time was 0 , that is after $1 \mathrm{~h}$ soaking but before any heating had occurred, a large amount of water (the cooking water) surrounded the rice grains. Small cracks were observed in some of the Nipponbare (Fig. 2) and Khao Dawk Mali (Fig. 3) rice grains, but not in the High Amylose grains (Fig. 4). Although the rice grains appear almost completely black at this point they did contain some water (at least the $0.15 \mathrm{~g} / \mathrm{g}$ present in the raw, unsoaked rice). Signals from this water were not detected because the $\mathrm{H}_{2} \mathrm{O}$ molecules have very restricted mobility at these low concentrations and temperatures. Between 0 and 8 min cooking, no obvious changes were observed for any of three types of rice.

When the cooking time was $10 \mathrm{~min}$ (the sample temperature was around $80^{\circ} \mathrm{C}$ at this time point), the Nipponbare grains had obvious fissures, but the number of cracks was limited to a few per grain and the grains retained their shape. Water had entered the rice through all surfaces which were in direct contact with the cooking water but a large proportion of the grain fragments remained dark. When the cooking time was $12 \mathrm{~min}$, the sample temperatures were around boiling $\left(100{ }^{\circ} \mathrm{C}\right)$ and by this time the Nipponbare grains had absorbed a significant amount of water and swelled slightly. Liquid cooking water was still present between the grains and there were still some dark gray regions in the images indicating that the absorbed water was not evenly 
distributed throughout the grains.

After 15 min of cooking, the Nipponbare grains had absorbed more water and expanded enough to touch one another, but it was still possible to discern boundaries between the individual grains. The black areas near the center of the image show there were empty spaces between the grains which indicates that all of the cooking water had been absorbed. The graduations in the grayscale intensities seen within the rice grains reveal that the water was not evenly distributed. After cooking for $20 \mathrm{~min}$ the water appeared to be distributed more uniformly within the grains, and by $30 \mathrm{~min}$ it appeared to be very homogeneously distributed. The boundaries between individual grains, barely visible at $20 \mathrm{~min}$, had completely vanished after cooking for a further $10 \mathrm{~min}$.

For the Khao Dawk Mali rice, very extensive cracking had occurred by 10 min and a significant amount of water had entered the grains from all of the exposed surfaces. It is not clear from these images whether cracking preceded water absorption or if rapid water uptake caused the grains to crack. After $12 \mathrm{~min}$, the fragmented grains had absorbed all of the cooking water (empty spaces between the grains are observed) and swelled to the extent that they merged together. This swelling may indicate that the starch granules had started to rupture and become gelatinous. The water distribution within the Khao Dawk Mali was not yet completely homogeneous but was more uniform than observed for Nipponbare rice. By $15 \mathrm{~min}$, the water within individual grains had become more evenly distributed, but no obvious changes in the morphology of the grains were seen. The overall brightening of the pixels may indicate the onset of changes in the molecular structure as the starch begins to reach a cooked state.

Very elongated Khao Dawk Mali grains were seen after 20 min cooking. The small dark gray regions are interpreted as void spaces between sections of the fragmented grains that were previously filled with cooking water. Based on the image acquired after 10 min of cooking (which clearly shows cracks passing completely through most grains), it is likely that the fragmented grains have not disintegrated completely because they are resting on other grains. These supporting fragments are not seen because they are outside the 1-mm thick slice through the sample. The image acquired after 30 min showed some grains with uniform pixel intensities, and some with concentric contours of varying grayscale intensity. It is unlikely that these "rings" indicate uneven water contents within individual grains given the results observed after 20 min of cooking. A more plausible interpretation is that the rings result from averaging of different proportions of white (arising from the rice) and black (from the empty voids) within the 1-mm 
thick image slice.

For the High Amylose grains, it appears that little or no water absorption had occurred even after $10 \mathrm{~min}$ of heating and there is no indication that the grains had cracked. The grayscale image acquired after 12 min of cooking shows a slight brightening in the intensity pixels at the periphery of the individual grains which indicates that some water had been absorbed near the grain surfaces. There was no indication that significant quantities of water had penetrated to the interior of the High Amylose grains. At the 15 min time point, water uptake through the outer surfaces had continued but there were still large regions in the interior of each grain which did not contain significant amounts of water. After $20 \mathrm{~min}$, the High Amylose rice had absorbed more water through the outer surfaces, but the inner cores of the grains still had low water contents. The grains remained intact (no cracks observed), and retained their size and shape. By 30 min water had reached the innermost parts of the grains. Although the grains had swelled and changed shape, boundaries between the individual grains are still discernable.

\section{$3.2 T_{2}$ contour images}

The $T_{2}$ images for the three different kinds of rice are not shown here but are included as supplementary material. In these images the bulk cooking water (and water in the reference capillary) appears white because it has a long $T_{2}$ value. As the water to starch ratio increases inside the rice grain, the $T_{2}$ values for the protons increase. Variations in water content within different parts of the rice grains are easier to detect in the $T_{2}$ images than in the grayscale first-echo images because the red coloration of the pixels having high water contents are more readily differentiated from the blue or black pixels corresponding to low water contents.

From the $T_{2}$ images, it is clear that all of the cooking water had been completely absorbed between 12 and 15 min of cooking for the Nipponbare rice. Khao Dawk Mali took less than 12 min, while more than 20 min of heating was required for this to occur in the High Amylose rice. Application of heat for a longer time resulted in progressively higher water contents, initially at the outer edges of the grains, and later in the interior regions. The cooking time needed to attain an almost homogenous distribution of $T_{2}$ values throughout the rice grains was the shortest for Khao Dawk Mali, followed by Nipponbare and then High Amylose. Although the actual $T_{2}$ values are not of interest for their own sake, conversion into quantitatively reliable water contents using the empirical calibration results in data having obvious practical value. 


\subsection{Water content and distribution}

The color images presented in the right-hand columns of Figs. 2-4 show the quantitatively reliable water content maps obtained for the three kinds of rice after various cooking times. In these images, black pixels represent water contents of less than $0.4 \mathrm{~g} / \mathrm{g}$ and increasing water contents are indicated by increasing color warmth from blue to red. Note that water in the reference capillary, and any residual cooking water which may have surrounded the rice, is not seen in these maps. Proton signals from this water were "edited-out" because the $T_{2}$ values are higher than the upper limit for eq 1. Black pixels in these maps therefore correspond to either regions having water contents less than $0.4 \mathrm{~g} / \mathrm{g}$ or to bulk (liquid) cooking water. Comparison of the color water content maps with the corresponding grayscale first-echo (or $T_{2}$ ) image allows the presence or absence of cooking water to be determined.

For Nipponbare rice (Fig. 2), the water content was below $0.4 \mathrm{~g} / \mathrm{g}$ for virtually all parts of the rice grains at cooking times shorter than $8 \mathrm{~min}$. By $10 \mathrm{~min}$, the water content had increased abruptly for those regions exposed directly to cooking water (i.e. both the original exterior of the grains and any surfaces exposed by cracking). The interiors of the grains had water contents of $0.5 \mathrm{~g} / \mathrm{g}$ or less. By $12 \mathrm{~min}$, the majority of the rice sample had water contents between 0.6 and $0.7 \mathrm{~g} / \mathrm{g}$ although the interior regions had lower contents and the grain peripheries had higher contents. After $15 \mathrm{~min}$, the water content within the sample as a whole had become more homogeneous. As the cooking time increased from 20 to $30 \mathrm{~min}$ the proportion of the rice having a water content above $0.8 \mathrm{~g} / \mathrm{g}$ or below $0.7 \mathrm{~g} / \mathrm{g}$ decreased, while the fraction having water contents between 0.7 and $0.8 \mathrm{~g} / \mathrm{g}$ increased. Even after cooking for 30, min there were still some small portions of the sample with water contents below $0.6 \mathrm{~g} / \mathrm{g}$. This indicates that the water contents of the grains became progressively more homogenous with increasing time at $100{ }^{\circ} \mathrm{C}$, consistent with increasing gelatinization of the amylopectin-rich starch.

The water uptake and its distribution in the Khao Dawk Mali rice (Fig. 3) occurred significantly faster than for the Nipponbare rice, even though it too had water contents below 0.4 $\mathrm{g} / \mathrm{g}$ until some point between 8 and $10 \mathrm{~min}$. By $10 \mathrm{~min}$, the water content was higher than for Nipponbare rice at the same cooking time. This faster rate of water uptake was likely facilitated by the severe fragmentation that had occurred. By the onset of boiling, all of the cooking water had been absorbed and the water contents had reached 0.5 to $0.9 \mathrm{~g} / \mathrm{g}$. By $15 \mathrm{~min}$ the entire sample had reached a state of almost completely uniform water content, with the major portion of the sample containing between 0.7 and $0.8 \mathrm{~g} / \mathrm{g}$ of water. No obvious changes were seen as the 
cooking time was increased to $30 \mathrm{~min}$.

For High Amylose rice (Fig. 4), significant uptake of water did not occur until some point between 10 and 12 min of cooking. The water absorption progressed more slowly than for the other types of rice. The "rings" of color seen in the water content maps indicate that the highest water contents occurred nearest the grain surfaces and that the water concentration decreased with increasing distance from the outer surfaces. After 20 min of heating the water content within the center of almost half of the grains was still $0.4 \mathrm{~g} / \mathrm{g}$ or below, less than $0.7 \mathrm{~g} / \mathrm{g}$ in the remainder, and water was still present outside the grains. By 30 min, all of the cooking water had been absorbed, and although the water had become more uniformly distributed within the individual grains, the water content was still higher at the outer edges of the grains than in the interiors.

Fig. 5 shows three-dimensional intensity plots of the water content for the three kinds of rice after cooking time for $15 \mathrm{~min}$. This alternative presentation format emphasizes differences in water contents and thereby facilitates comparisons between the samples. It can be seen that the water within the Nipponbare rice grains is less evenly distributed than in Khao Dawk Mali rice, which had the most homogenous water content of the three types of rice studied. The water within the High Amylose rice grains was clearly not uniformly distributed by this time point. The low water content in the interiors of the grains is obvious.

\subsection{Average water content and degree of cooking}

In addition to the specific water contents at different positions within the rice grains it is desirable to be able to measure and monitor changes in the average water content of the whole rice sample during the cooking process. Fig. 6 shows the average water contents determined for the three kinds of rice at various times during the cooking process. These averages were calculated from the water content data and included water present initially in the raw rice but excluded water that was outside the grains.

The average water content of Nipponbare rice did not increase from its initial value of $0.36 \mathrm{~g} / \mathrm{g}$ until heat had been applied for more than $8 \mathrm{~min}$. It can therefore be inferred that water uptake only began to increase significantly once the temperature was above $50{ }^{\circ} \mathrm{C}$. The water content increased between 8 and 12 min until it reached its maximum level of $0.73 \mathrm{~g} / \mathrm{g}$ and then leveled off. Water uptake appears to have stopped at, or slightly before, $15 \mathrm{~min}$. This is consistent with the complete absence of cooking water in the first echo image acquired at the same cooking 
time.

Very similar behavior was seen for the Khao Dawk Mali samples, although this rice reached a marginally higher average water content. Water absorption appeared to stop for the Khao Dawk Mali rice at or near the onset of boiling. This observation agrees with the absence of cooking water in the corresponding first echo image data.

Water uptake in High Amylose rice was not detectable until it had been heated for 8 to 10 min. The subsequent increase in average water content progressed significantly more slowly than for the other types of rice. Although the average water content after $30 \mathrm{~min}$ of cooking was close to $0.7 \mathrm{~g} / \mathrm{g}$, equilibrium had still not been reached. Based on the data presented in Fig. 6, more than twice the time was required for High Amylose rice to achieve an average water content of $0.7 \mathrm{~g} / \mathrm{g}$ compared to Nipponbare or Khao Dawk Mali rice.

The average water contents of Nipponbare, Khao Dawk Mali and High Amylose rice after cooking, as determined from the NMR imaging data, were $0.72,0.74$, and $0.71 \mathrm{~g} / \mathrm{g}$ respectively. The theoretically predicted water contents were $0.71,0.70$, and $0.70 \mathrm{~g} / \mathrm{g}$, respectively. The good agreement with the predicted values confirms that the water has been quantitatively detected.

The degree of gelatinization of the starch is a major factor determining whether the rice is considered to be fully cooked. The degree of cooking can be deduced from the localized water content. Fig. 7 shows the percentages of each cooked state for the three types of rice at various times after heating began determined in this way. The fraction labeled " $T_{2}$ outside range" corresponds to pixels with little or no proton signal and therefore $T_{2}$ values outside the valid ranges of eq 1 . For all three rice types the percentage of the rice with invalid $T_{2}$ values decreased quickly once water uptake began.

As expected, the fraction of the sample in the optimally-cooked state increased for all three types of rice as the cooking time progressed. It took 30 min of cooking for approximately $90 \%$ of the Nipponbare rice to reach the optimally-cooked state while the Khao Dawk Mali rice reached this same percentage of optimally-cooked rice after $20 \mathrm{~min}$. The fraction of the Nipponbare and Khao Dawk Mali samples which was over-cooked tended to decrease with increasing cooking time after $10 \mathrm{~min}$. This indicates that the initially high water contents near the outer surfaces were reduced as the water migrated into the interior of the grains.

The High Amylose rise showed a much slower increase in the percentage of optimally-cooked rice with increasing heating duration, and this fraction reached a maximum of $70 \%$ after cooking for $30 \mathrm{~min}$. The percentage of over-cooked rice increased slightly with 
increasing cooking time, and the water diffusion and starch gelatinization seem to be interdependent.

The maximum fractions of under-cooked rice for both Nipponbare and Khao Dawk Mali rice were observed after $10 \mathrm{~min}$ of heating, and at $15 \mathrm{~min}$ for the High Amylose rice. The behavior of this fraction in conjunction with the amount of over-cooked rice may provide insight into the differences observed in the rates of water uptake and diffusion for the different types of rice.

The degree of interaction between the water and the starch molecules in the rice grains will depend on the water concentration, the temperature, the heating duration, and the starch gelatinization temperature. Differences in the composition of the starch will result in different gelatinization temperatures. After water is absorbed it must diffuse to the interior of the grain before complete cooking can occur. Although water diffusion is usually promoted at higher temperatures, reactions with the starch molecules will compete for the water molecules and may thus slow or hinder diffusion.

Assuming that the molecular mechanisms for both water absorption and diffusion are the same for all types of rice, the experimental data presented here suggest that higher amylose contents slow water diffusion. For rice types having similar amylose contents, diffusion of water and starch gelatinization occurred faster in Khao Dawk Mali (Indica, long grain) than in Nipponbare (Japonica, short grain) which indicates that other components present in the rice may also affect these processes. It is also proposed that differences observed in the rate of water uptake, diffusion and distribution correlate to the known differences in the texture and cooking times. Although the hardness of cooked Nipponbare and Khao Dawk Mali rice is almost the same, the stickiness is not. These results suggest that the characteristic texture (stickiness) of Japonica rice is not related solely to the ease of water absorption. Further studies to investigate the underlying causes of these differences are underway. 


\section{Conclusions}

NMR imaging experiments have revealed that water absorption and diffusion occurred at different rates in Nipponbare, Khao Dawk Mali and High Amylose rice during cooking. Significant water uptake did not occur for any of the three types of rice until the cooking temperature was above $40{ }^{\circ} \mathrm{C}$. Once water absorption began it occurred more rapidly for Khao Dawk Mali than for Nipponbare rice. For the High Amylose grains the rate of water uptake was less than half that seen for the other types of rice. The diffusion of water molecules within the High Amylose rice also occurred much more slowly than in Nipponbare and Khao Dawk Mali rice. Cooking may have caused the Khao Dawk Mali grains to fragment, while High Amylose grains remained intact throughout the cooking process.

The NMR imaging experiments used in the present study made it possible to detect and quantify the processes of water uptake and water diffusion during the cooking process of all three types of rice. The local and average water contents, as well as the degree of cooking, were also measured. The data obtained are quantitatively reliable and can be interpreted in a straightforward way. The various images and graphs provide detailed information about the location and state of the water molecules, much of which could not be readily obtained by other methods. It should be possible to gain a better understanding of the optimal cooking conditions for other types of rice using this protocol. 


\section{References}

Horigane, A. K., Toyoshima, H., Hemmi, H., Engelaar, W. M. H. G., Okubo, A., \& Nagata, T. (1999). Internal hollows in cooked rice grains (Oryza sativa cv. Koshihikari) observed by NMR micro imaging. Journal of Food Science, 64, 1-5.

Juliano, B. O., Perez, C. M., Blakeney, A. B., Castillo, T., Kogseree, N., Laignelet, B., Lapis, E. T., Murty, V. V. S., Paule, C. M. \& Webb, B. D. (1981). International cooperative testing on the amylose content of milled rice. Starch / Stärk, 33, 157-162.

Kasai, M., Lewis, A., Marica, F., Ayabe, S., Hatae, K. \& Fyfe, C. A. (2005). NMR imaging investigation of rice cooking. Food Research International, 38, 403-410.

Mohoric, A., Vergeldt, F., Gerkema, E., Jager, A., Duynhoven, J., Dalen, G. \& As, H. (2004). Magnetic resonance imaging of single rice kernels during cooking. Journal of Magnetic Resonance, 171, 157-162

Nakazawa, F., Noguchi, S., Kato, M., Takahashi, J., \& Katada, T. (1978). Water binding of cooked rice determined by pulsed NMR. Journal of Home Economics Japan, 29, 140-143.

Nishimura, K., Goto, M., Imazuya, N., Nakai, S. (1997). Optimum cooking conditions for Indica type rice using random centroid optimization. Journal of Cookery Science of Japan, 30, 9-16.

Origin. Version 7.0 OriginLab Corporation. One Roundhouse Plaza, Northampton, MA 01060 USA

Paravision. (1996a). GEFI Method. Paravision user manual (p. 981). Version 2.1. Germany: Bruker Medical GMBH.

Paravision. (1996b). MSME Method. Paravision user manual (p. 983). Version 2.1. Germany: Bruker Medical GMBH.

Paravision. (1996c). User manual. Version 2.1. Germany: Bruker Medical GMBH. 
2 Takeuchi, S., Fukuoka, M., Gomi, Y., Maeda, M., \& Watanabe, H. (1997a). An application of 3 magnetic resonance imaging to the real time measurement of the change of moisture profile in a 4 rice grain during boiling. Journal of Food and Engineering, 33, 181-192.

5

6 Takeuchi, S., Maeda, M., Gomi, Y., Fukuoka, M., \& Watanabe, H. (1997b). The change of 7 moisture distribution in a rice grain during boiling as observed by NMR imaging. Journal of 8 Food and Engineering, 33, 281-297.

9 


\section{Figure Captions}

Fig. 1. Photographs of the three different types of rice investigated by NMR imaging. a) Nipponbare (Japonica), b) Khao Dawk Mali (Indica), and c) High Amylose (Indica) rice.

Fig. 2. First echo images and water concentration distributions calculated from the ${ }^{1} \mathrm{H} T_{2}$ NMR images for Nipponbare rice at the indicated cooking times (in minutes).

Fig. 3. First echo images and water concentration distributions calculated from the ${ }^{1} \mathrm{H} T_{2} \mathrm{NMR}$ images for Khao Dawk Mali rice at the indicated cooking times (in minutes).

Fig. 4. First echo images and water concentration distributions calculated from the ${ }^{1} \mathrm{H} T_{2}$ NMR images for High Amylose rice at the indicated cooking times (in minutes).

Fig. 5. 3-D presentation of water content distribution within the different types of rice after 15 min cooking. a) Nipponbare rice, b) Khao Dawk Mali rice, c) High Amylose rice.

Fig. 6. Changes in average water content for the different rice types during cooking.

Fig. 7. Percentage of rice in each cooking state for the different rice types as a function of cooking time. a) Nipponbare rice, b) Khao Dawk Mali rice, c) High Amylose rice. 
Figure 1

Click here to download high resolution image
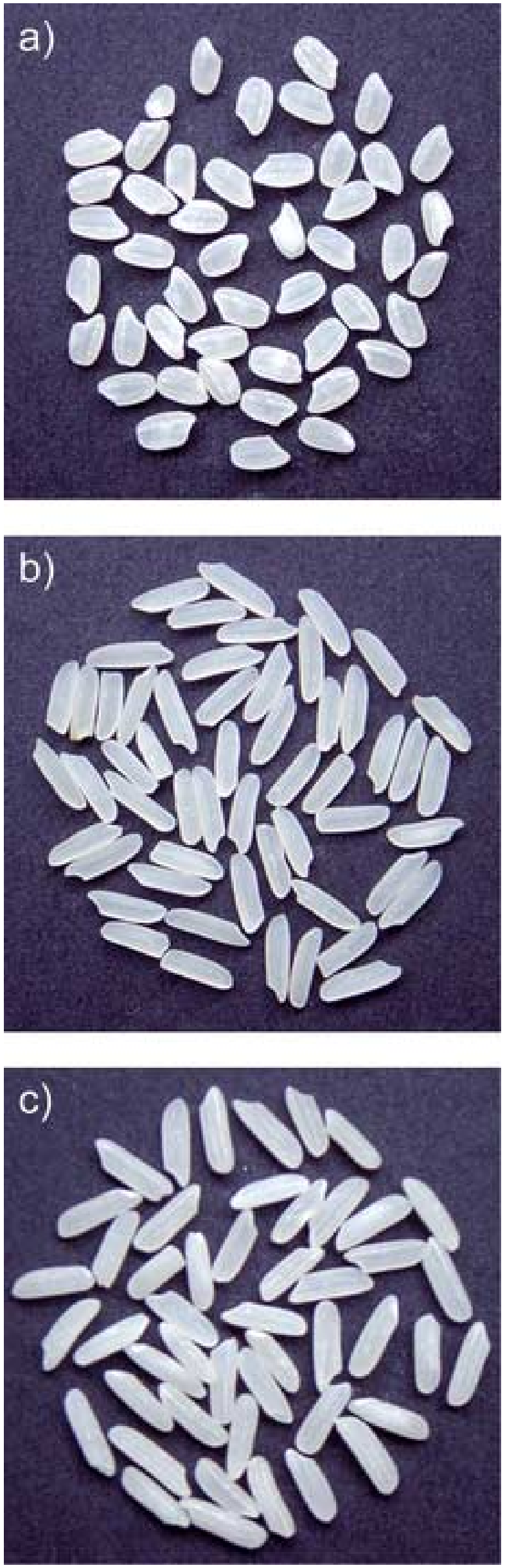
Figure 2

Click here to download high resolution image
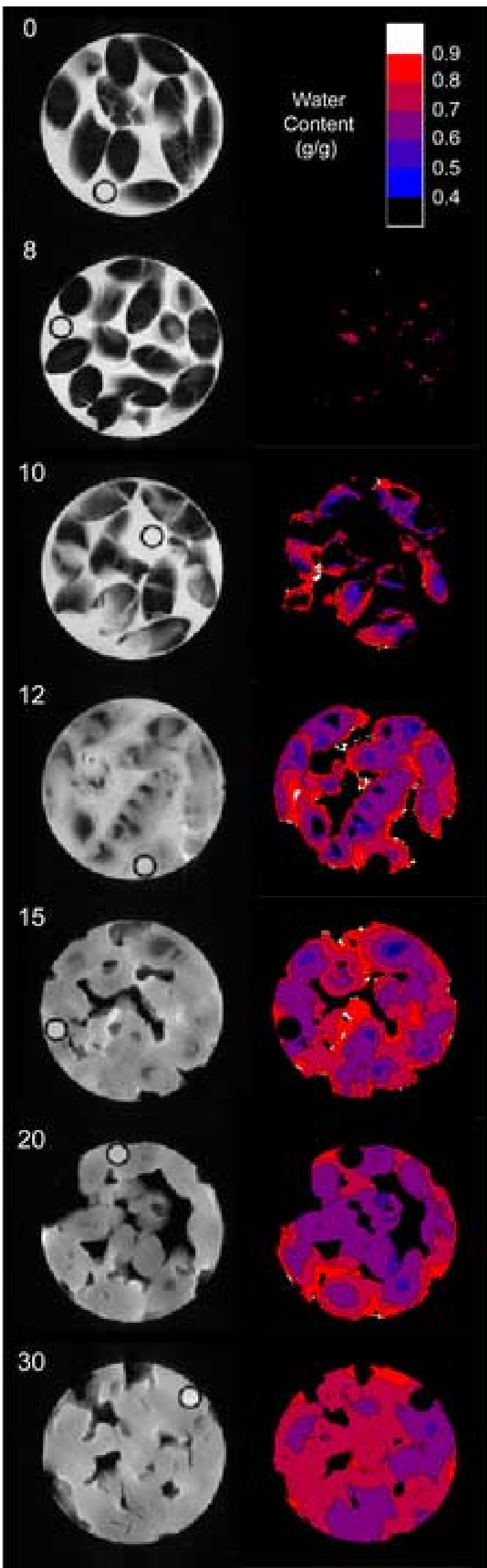
Figure 3

Click here to download high resolution image

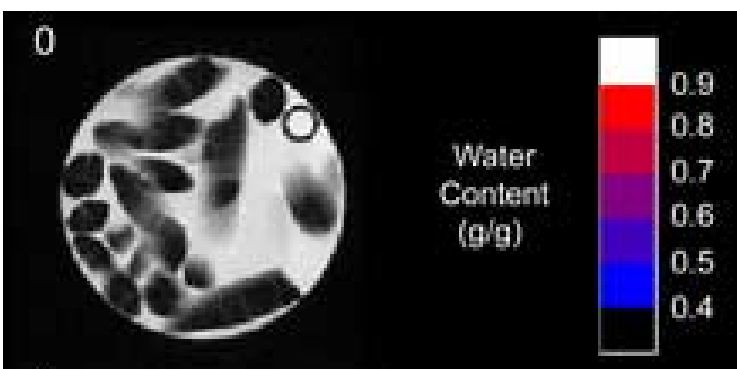

8

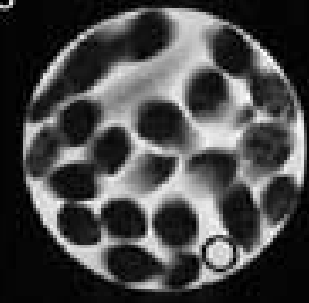

10

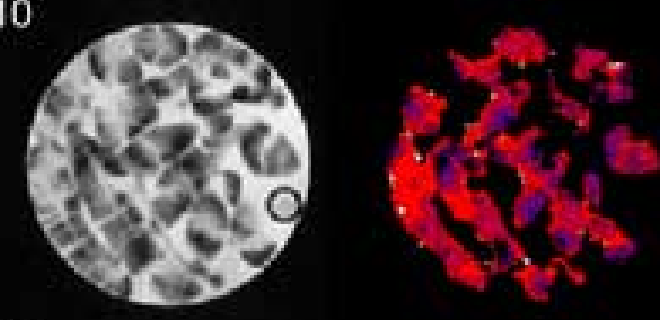

12

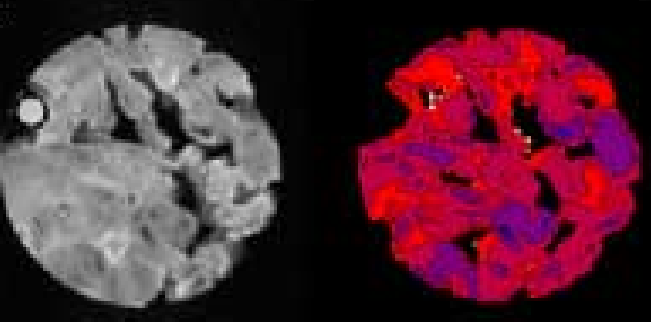

15
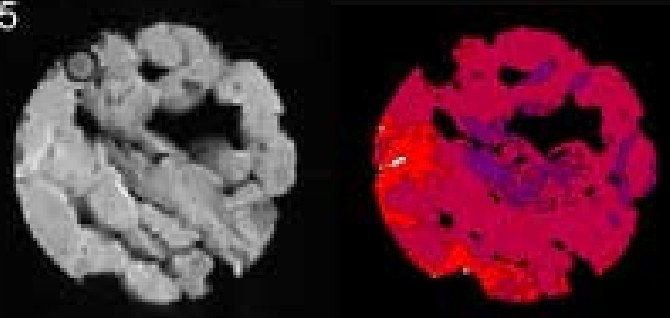

20

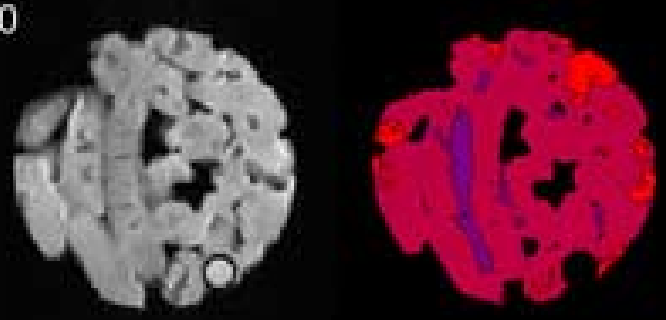

30

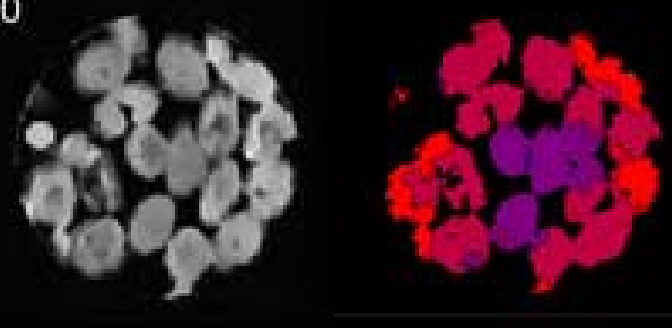


Figure 4

Click here to download high resolution image

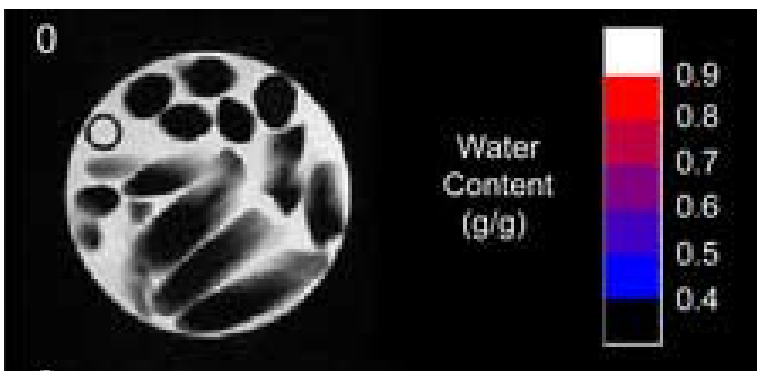

8

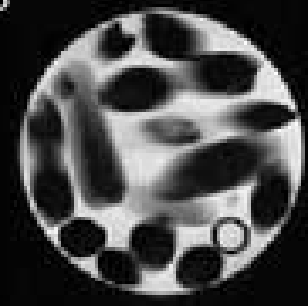

10

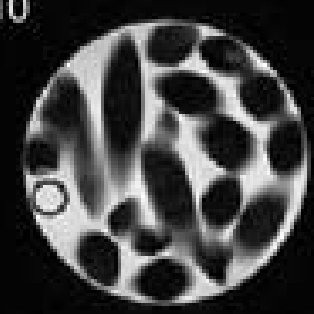

12

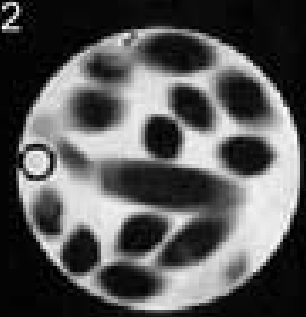

15
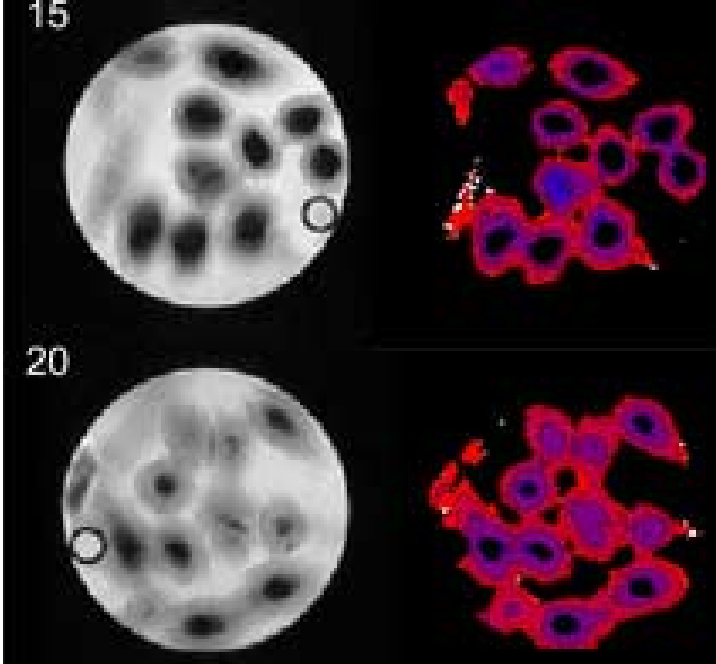

30
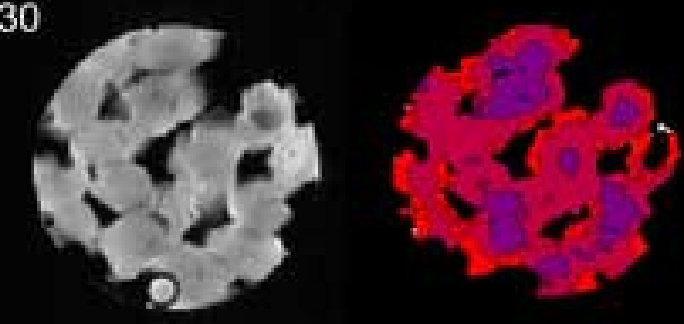
Figure 5

Click here to download high resolution image
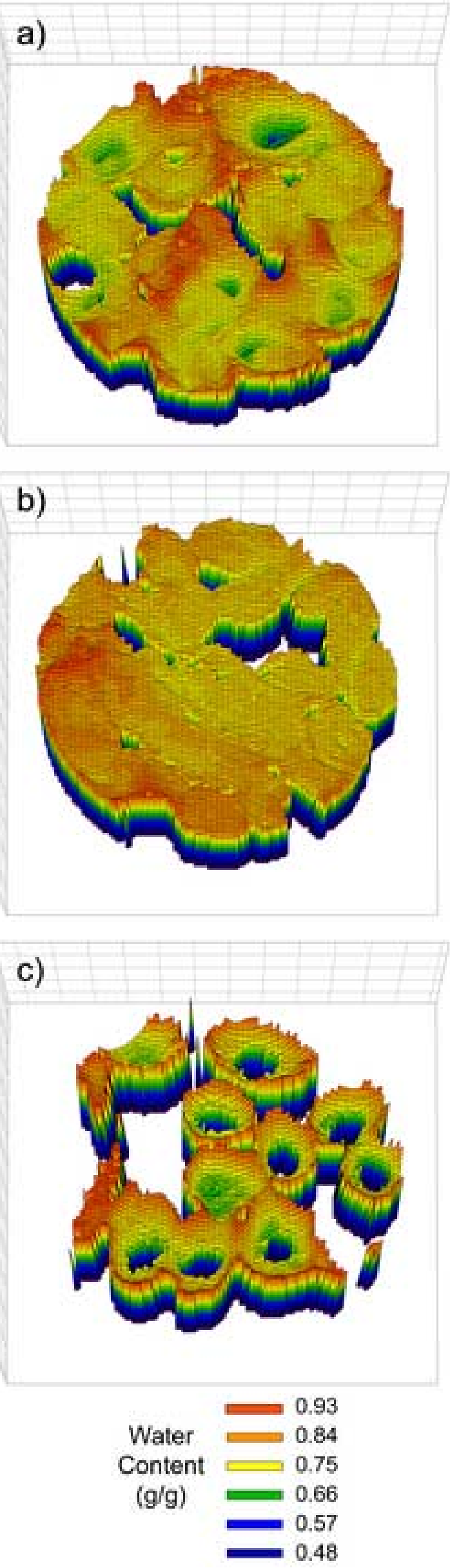


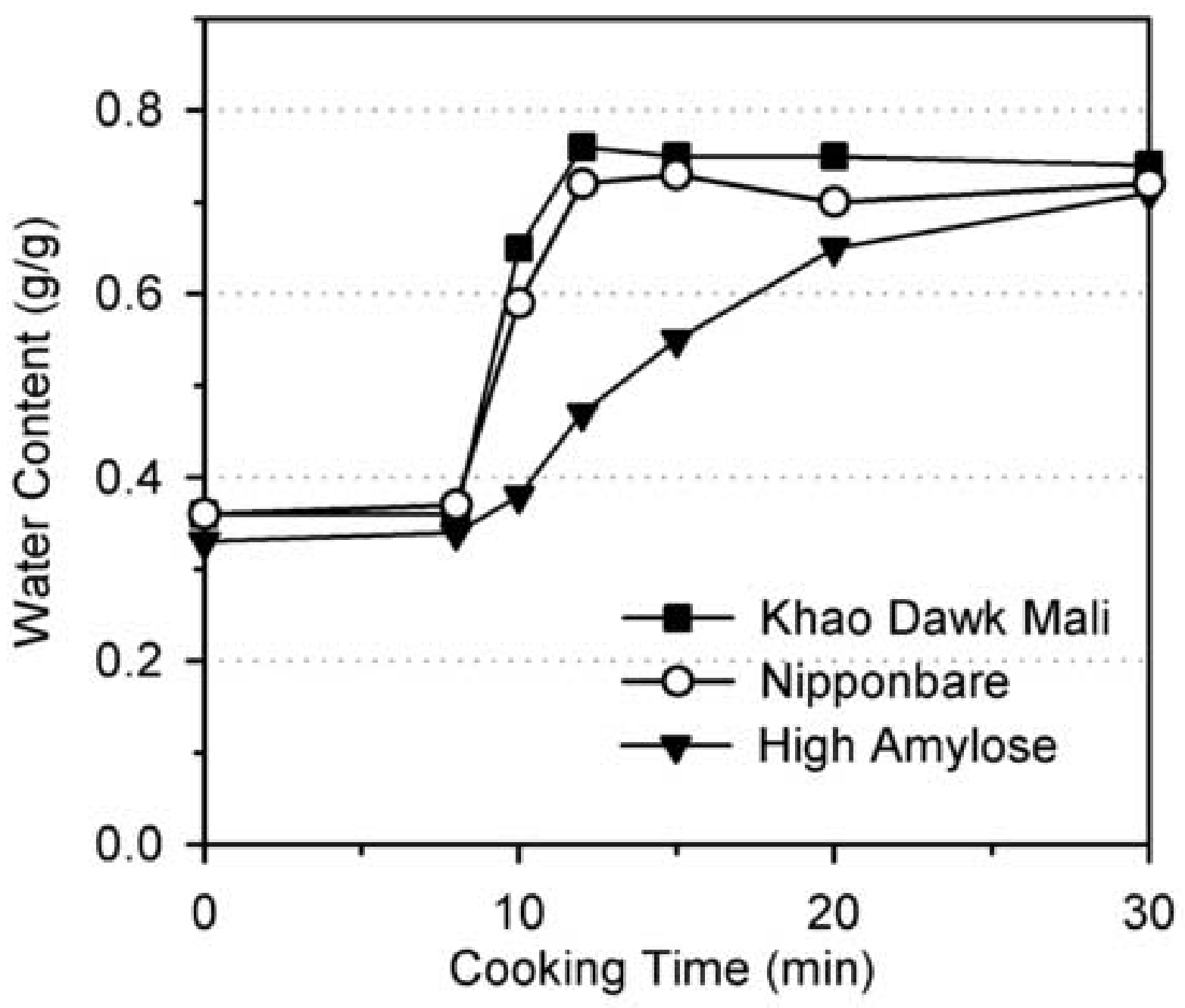


Click here to download high resolution image

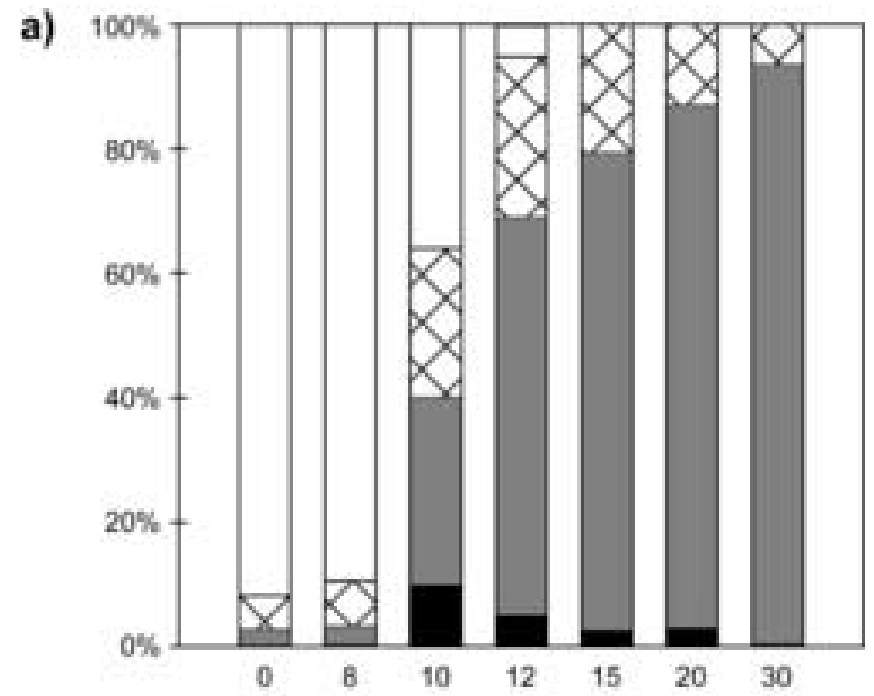

b)

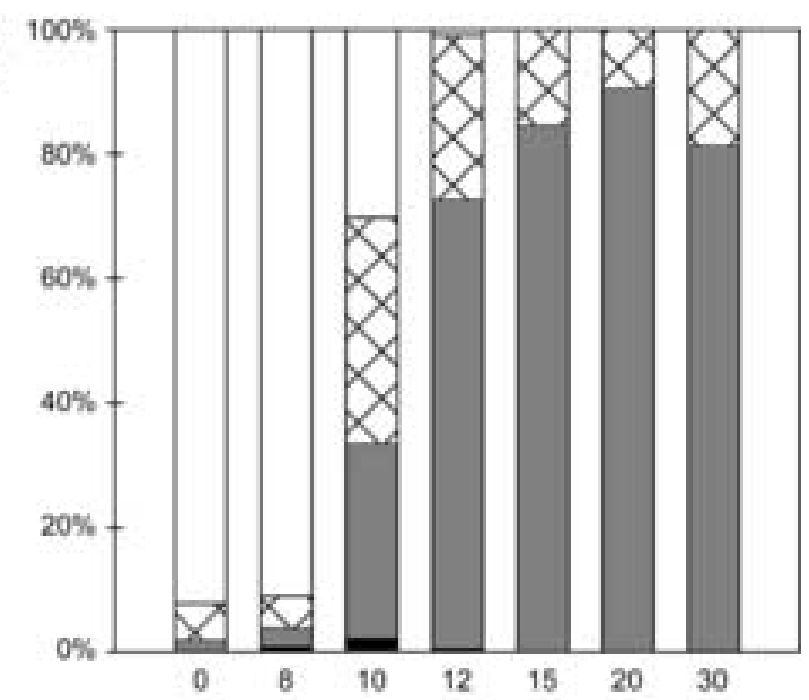

c)

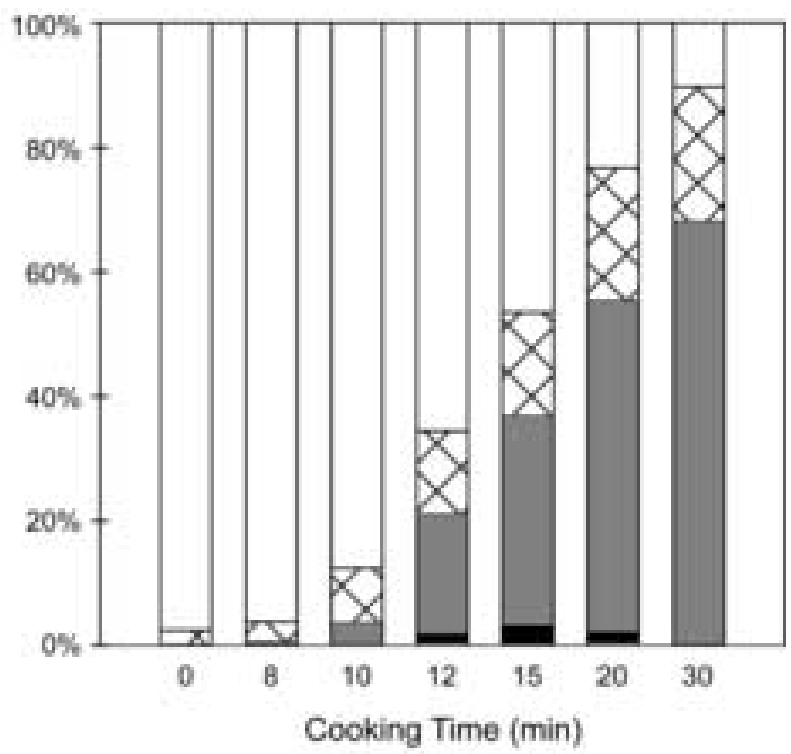

$\begin{array}{ll}\square \text { under-cooked } & \square \text { cptimally-coobed } \\ \square \text { over-cooked } & \square T_{2} \text { outside range }\end{array}$ 


\section{Supplementary Information (Figure S1)}

Kasai et al. Quantitative NMR imaging study of the cooking of Japonica and Indica rice - Supplementary Material

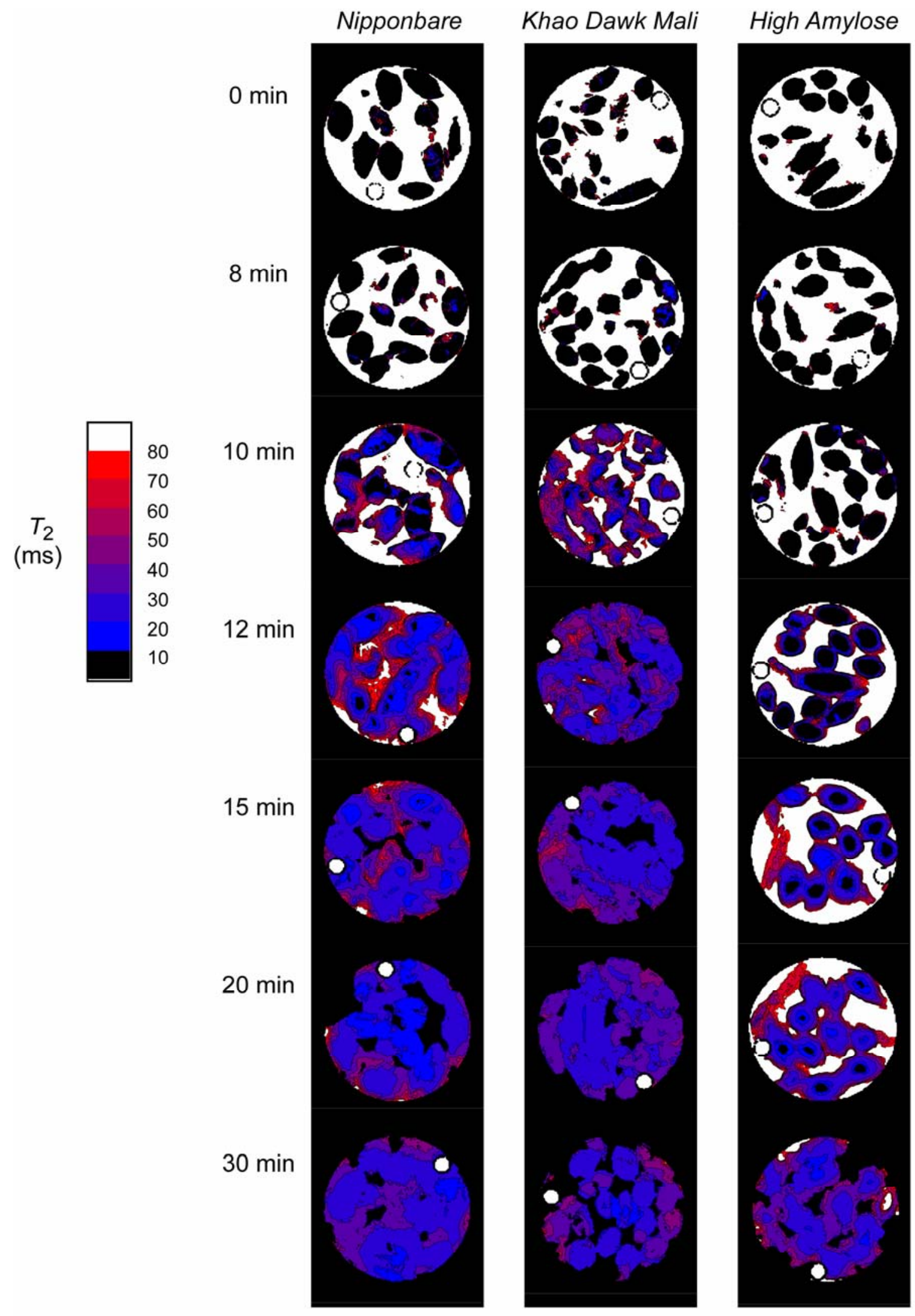

Fig. S1. Contour representation of ${ }^{1} \mathrm{H} T_{2}$ images for three different types of rice at the cooking times indicated. Images were calculated by fitting the intensities in the first 16 echoes of the corresponding MSME experiments. Small white circles are glass capillary tubes filled with distilled water used as a calibration reference. 